\title{
A Social Network Model of Direct versus Indirect Reciprocity in a Corrections-Based Therapeutic Community
}

Nathan Doogan, Keith Warren, Danielle Hiance, Jessica Linley

The Ohio State University

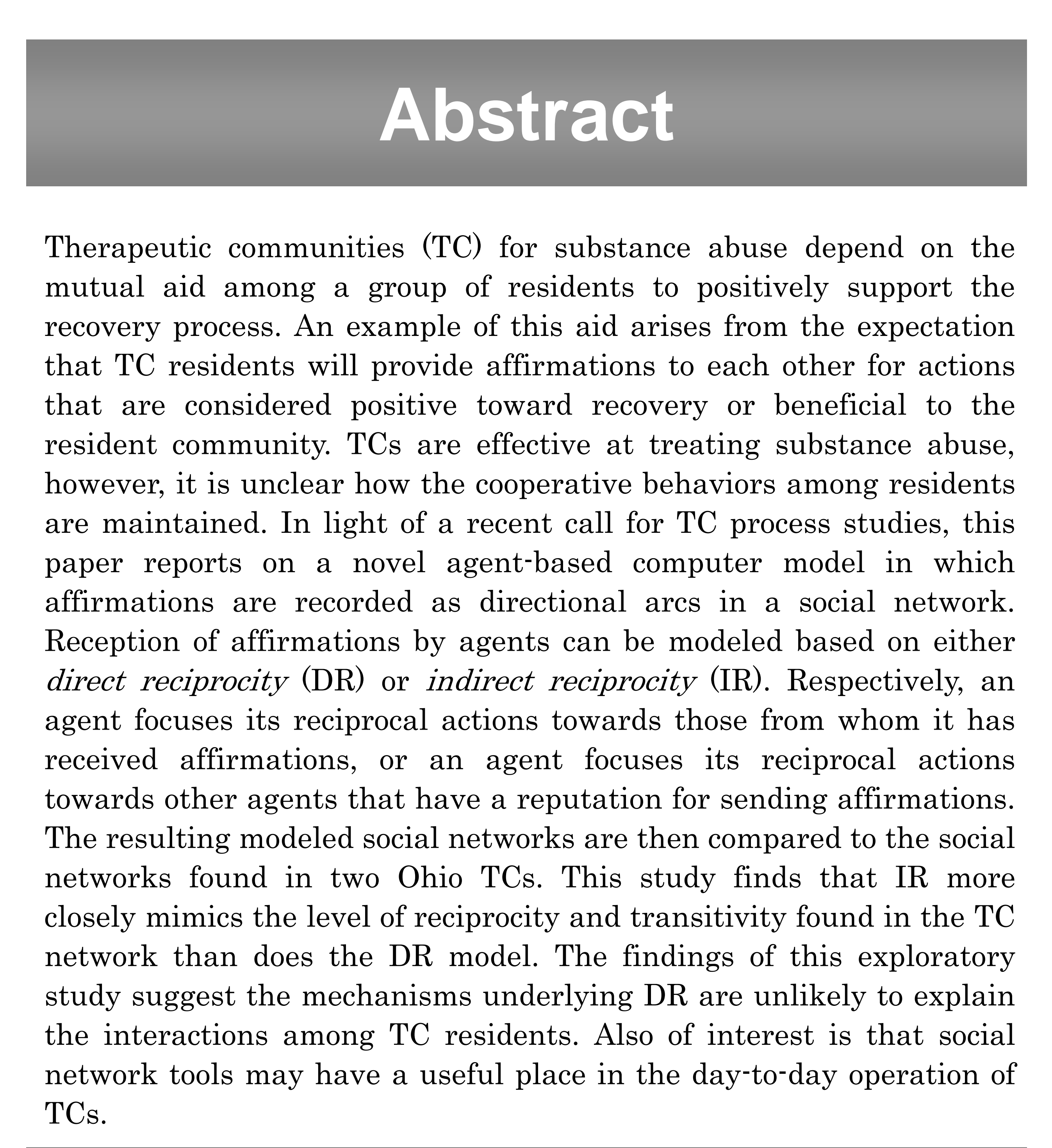

\section{Introduction}

Residential therapeutic communities $(\mathrm{TC})$ are the most common form of
system.

-Such TCs house as many as 150 residents at one time.

-TCs are based on mutual aid between residents, who are expected to support each other in recovering from substance abuse,

$-T C$ researchers have gone so far as to say that the
fellow substance abusers is the method of treatment.

-Qualitative studies have suggested that TC residents do value the help of their peers,
culture of mutual aid.

-At this point there has been no quantitative study of the
maintenance of cooperation in a TC.

- This study aims to address the lack of research using a social
network perspective of resident interactions to explore the network perspective of resident interactions to explore the
mechanisms supporting cooperation among the residents of a TC.

\section{Theory}

GAME THEORETIC MODELS OF COOPERATION IN GROUPS Direct Reciprocity: In a computer-based simulation tournament,
Axelrod and Hamilton (1981) found that a tit-for-tat model of direct reciprocity was able to produce cooperation in groups if the chances of
meeting another player more than once during a multi-round game is

will cooperate with peers with whom I have experienced
cooperation before.

\section{Theory (continued)}

Indirect Reciprocity: Consists of two subtly distinct phenomena Upstream: If someone scratches my back, I will be more likely to scratch someone's back (pay it forward).
Downstream: If you scratch someone's back, I will be more likely to
scratch your back (reputation).

Martin Nowak (2005) compares direct reciprocity to barter. Downstream shopping sites. The number of online stores is so large that relationship between an average buyer and the store is unlikely. But I
will buy from a particular site if I am sufficiently convinced by their will buy from a particular site if $\mathrm{I}$ am
reputation that I am not going to get taken.

DIRECT AND INDIRECT RECIPROCITY IN THE TC

TC research suggests that upstream indirect reciprocity is a relevant construct in the study of TCs as affirir
residents to help others (DeLeon, 2000).

It is less clear what role direct and indirect reciprocity play in the
cooperative behaviors of residents in a TC. Affirming good behavior could be thought of as a risky venture among this population. A rejection could well to bave arior relationship with anosthon hefat a resident would do do This matches the model of direct reciprocity.

On the other hand, TC theory suggests that role models ought to be held in high regards in the community. Therefore, staff encourage and reward
affirmative messages among residents. Additionally, the senders and receivers of affirmative messages are often announced at meal times in
the TC. Such activity could potentially lead to a reputation for helpful
behaviors. This matches the model of reputation - indirect reciprocity.

Method
AGENT BASED MODEL

For this study, two agent-based models (ABM) are developed to simulate
the sending and reception of affirmations in a TC: one based on direc reciprocity $(D R)$, the other based on downstream indirect reciprocity $(I R)$ (reputation). Both models utilize upstream indirect reciprocity to model
the likelihood of an agent sending an affirmation. DR and IR are the the likelihood of an agent sending an affirmation. DR and IR are the bases this choice on previous interactions. IR bases this choice on the
bection Direct Reciprocity (DR)

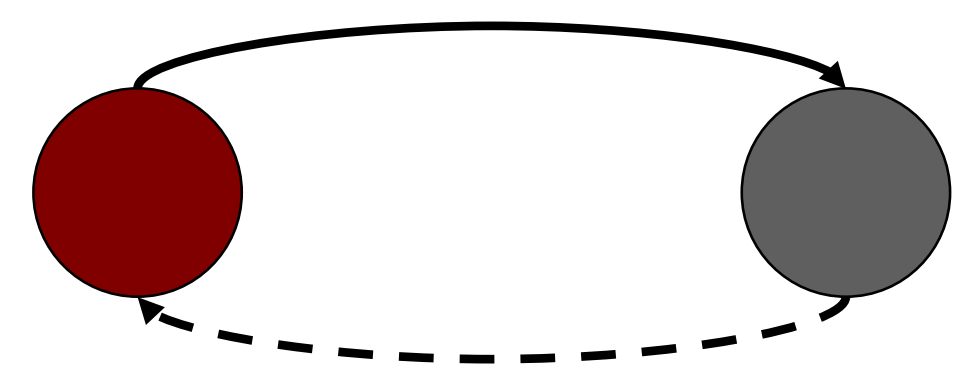

In the DR model, agents keep a list of other agents from whom they have received an a alimmation. Agents select a rectiver from this list Each agent agent being chosen drops.

Indirect Reciprocity (IR)

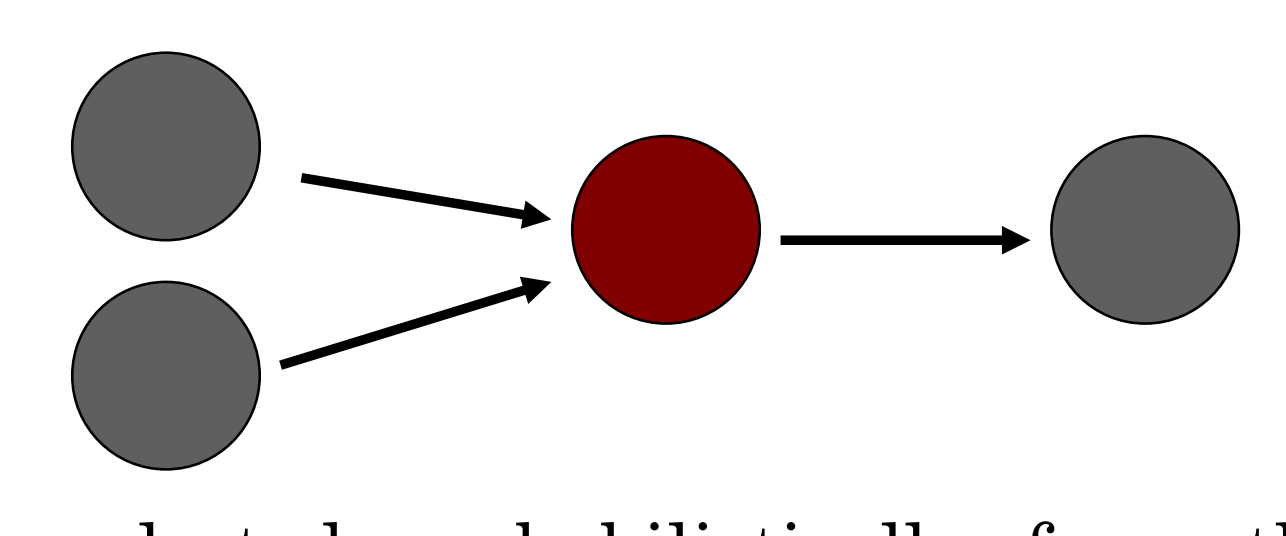

Affirmation receivers are selected probabilistically from the entire popul.

Both models are run over many iterations of one of these two receiver
Method (continued) ANALYSIS

Data: Data of sent affirmations has been captured from real resident interactions in two Ohio TCs (NW and WC). Affirmations in the TCs are
recorded and vetted by staff and residents for sincerity. The IDs of recorded and vetted by staff and residents for sincerity. The $1 \mathrm{Ds}$ of
affirmation senders and receivers are stored in a database. These records ed as a social network.

The ABMs also keep track of the IDs of sending and receiving agents. This also be represented as a social network

Model Fitting: ABM parameters are tuned to known values of network size reception of an affirmation has on the motivation of the receiving agent to
send an affirmation is the only parameter that was varied to fit the models send an affirmation
to empirical data.

The social networks produced by the TC ABMs and by the residents of the
real TC are compared on four social network measures for similarity - two real $\mathrm{TC}$ are compared on four social network measures for similarity - two Transitivity is a measure of network closure that can be regarded as a
measure of agreement by residents about who should be affirmed. Triangle completion is another measure of network closure that implies indirectly

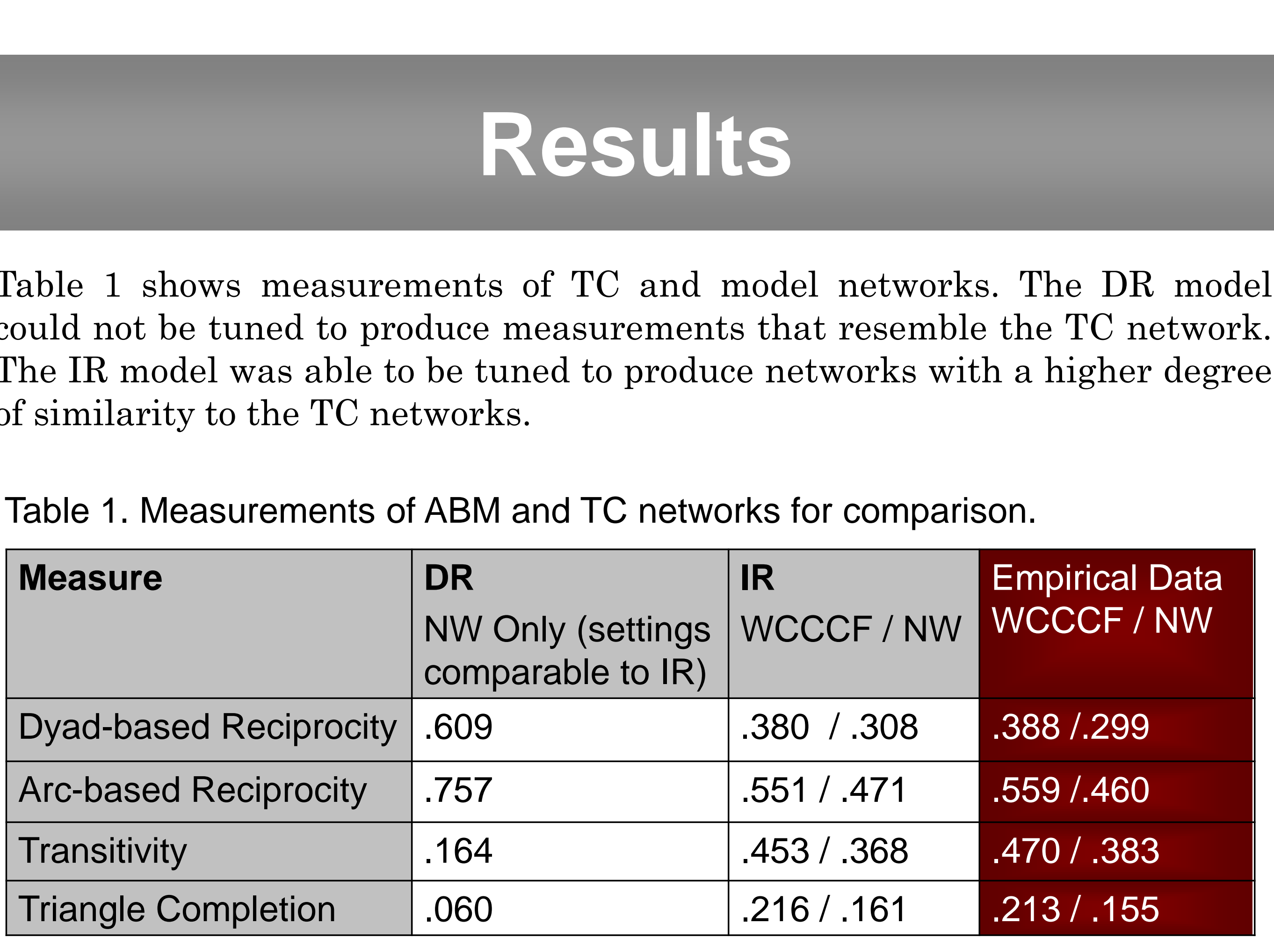

Table 2. Measurements of DR ABM networks given a sweep of the affirmation reception effect size parameter. NW TC network measurements included for

\begin{tabular}{|l|c|c|c|c|c|c|}
\cline { 2 - 7 } \multicolumn{1}{c|}{ Affirmation Reception Effect } \\
Network Measures & $\mathbf{0 . 5 0}$ & $\mathbf{0 . 9 0}$ & 1.10 & $\mathbf{1 . 5 0}$ & $\mathbf{2 . 0 0}$ & $\begin{array}{c}\text { Empirical } \\
\text { NW }\end{array}$ \\
\hline Dyad Reciprocity & 0.622 & 0.593 & 0.609 & 0.717 & 0.755 & 0.299 \\
\hline Arc Reciprocity & 0.767 & 0.745 & 0.757 & 0.835 & 0.860 & 0.460 \\
\hline Transitivity & 0.169 & 0.170 & 0.164 & 0.141 & 0.134 & 0.383 \\
\hline Triangle Completion & 0.062 & 0.062 & 0.060 & 0.051 & 0.049 & 0.155 \\
\hline
\end{tabular}

These tables indicate that the DR model tends too strongly towards reciprocal ties to match the $T C$ data regardless of parameter settings,
Relative to the $I R$ model, the $D R$ model produces far too many directly reciprocated ties and far too few transitive triads and completed triangles.
The IR model, on the other hand, appears to match the TC data much more

\section{Discussion}

The preceding analysis is exploratory

Only two empirical networks examined

-Statistics are descriptive network stats

-Reports are of individual simulations (i.e. no probability tests). not clear that the IR mechanism is analogous to the mechanism

The models are likely to be too simple to capture the effects of diversity among the residents in the TCs or appropriately capture
changes in individual behaviors that are theorized by TC
researchers.

Important to note about model fitting

The DR and IR models were not programmed to produce a certain
number of triads or reciprocal dyads. The results of these global measurements arose from local interactions of agents that are based

-Despite limitations, it appears direct reciprocity is not likely to be the only consideration at the individual level regarding who a resident will ferm. in the TC.

- This has some implications regarding the daily activities in the TC

- Perhaps reputation through indirect means - such as being nnouncement at lunch-time - plays a role in resident decisions gat practitioners should be aware of this as. To researchers and pra
unfolds.

-Future analysis of social networks in TCs might involve a series around the parameter values, thus allowing hypothesis testing to be completed.

- Alternative methods of analysis could include ERGM / P* evolution of the social network over time with considerations for Individual diversity among the actors in the network. These simplifying the process of developing an appropriate model to fit the data.

Understanding what makes one $\mathrm{TC}$ more effective than another has important

While these results can not speak directly to what makes one TC sudy to begin. The aforementioned methods, given appropriate

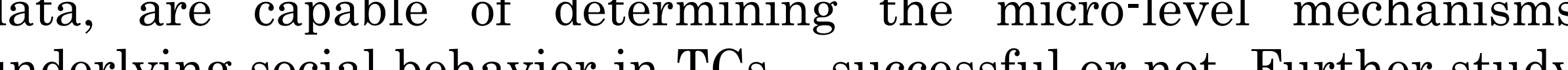
underlying social behavior in TCS - successful or not. Further study
of the social, political, and physical components of TSC that lead to
such mechanisms would make a revolution in the understanding of 